\title{
Diffusive Motion in Stage-1 and Stage-2 Li-Graphite Intercalation Compounds: Results of $\beta$-NMR and Quasielastic Neutron Scattering
}

\author{
A. Schirmer* and P. Heitjans \\ Institut für Physikalische Chemie und Elektrochemie, Universität Hannover, Callinstrasse 3-3a, \\ D-30167 Hannover
}

Z. Naturforsch. 50a, 643-652 (1995); received November 3, 1994

Dedicated to Professor Werner Müller-Warmuth on the occasion of his 65th birthday

Investigations of $\mathrm{Li}$ diffusion in stage- 1 and stage- $2 \mathrm{Li}$ graphite intercalation compounds with neutron time-of-flight and backscattering techniques and $\beta$-radiation detected nuclear magnetic resonance/relaxation $\left(\beta\right.$-NMR) with the nucleus ${ }^{8} \mathrm{Li}\left(T_{1 / 2}=0.8 \mathrm{~s}\right)$ are reviewed and compared. Depending on temperature, the spin-lattice relaxation-rate $T_{1}^{-1}$ of ${ }^{8} \mathrm{Li}$ is governed by different processes. Above $300 \mathrm{~K}, T_{1}^{-1}(T)$ shows maxima induced by long-range $\mathrm{Li}^{+}$diffusion. Jump correlation times are estimated. Inspection of the $B$ field dependence of $T_{1}^{-1}$ revealed two-dimensional diffusion behaviour. The neutron spectra showed a quasielastic line broadening above $500 \mathrm{~K}$, which was used to obtain diffusion coefficients and to trace jump vectors of the in-plane motion. The diffusion parameters observed with both techniques are compared, and differences that show up are discussed. In addition, the low-temperature spin-lattice relaxation rates, being due to coupling to conduction electrons, are used to explore electronic properties.

\section{Introduction}

The large variety of structures resulting from donor and acceptor insertion makes graphite intercalation compounds (GICs) one of the most extensively studied family of guest-host materials. Many properties of practical and theoretical interest, such as electronic transport and structure, ionic motion, magnetic structure and lattice dynamics, may be tailored by inserting appropriate guest species (the intercalate) into more or less well defined sites between the host layers. Additional variation derives from staging, i.e. the occupation of a periodic subset of the planes between the graphite layers. The number of graphite planes between the guest layers denotes the stage of the compound.

These features have stimulated a large part of the research activity dealing with GICs as model systems for studies of phase transitions, magnetism, electronic transport, ion dynamics etc. in two-dimensional (2D) systems, and comprehensive reviews are available now $[1,2]$.

Alkali-GICs are of particular interest since they provide simple guest species and exhibit nevertheless

\footnotetext{
* also at: IFF, Forschungszentrum Jülich.

Reprint requests to Dr. A. Schirmer.
}

a variety of in-plane structures. These range from commensurate long-range ordered ones with every guest site occupied, to discommensurate and dilute high-stage compounds.

The Li-GICs are a peculiar system due to the existence of the ordered stage- 1 and stage- 2 compounds, $\mathrm{LiC}_{6}$ and $\mathrm{LiC}_{12}$ respectively, which differ only in the additional C-layer in the stage- 2 compound. In both cases the $\mathrm{Li}$ atoms form a $2 \mathrm{D}$ hexagonal $(\sqrt{3} \times \sqrt{3})$ $\mathrm{R} 30^{\circ}$ superstructure with direct registry, i.e. the $\mathrm{Li}$ atoms sit directly above each other. Comparison of $\mathrm{LiC}_{6}$ and $\mathrm{LiC}_{12}$ thus offers the possibility to study phenomena for different interlayer coupling; a difference in in-plane Li density has not to be considered. It should also be mentioned that $\mathrm{Li}_{x} \mathrm{C}_{6}$ compounds are used as anode materials for rechargeable Li-ion batteries [3].

The main issue addressed in this contribution is the diffusive motion of $\mathrm{Li}$ and its dimensionality. Considering the small size of the $\mathrm{Li}$ ion it has been argued that diffusion jumps across the graphene sheets cannot be ruled out a priori as in the case of the heavy alkalis [4]. The direct registry of the carbon layers would provide such a diffusion path through the hexagon centres. Here the investigation and comparison of the stage- 1 and stage- 2 compounds is related to the question whether such a deviation from pure $2 \mathrm{D}$ 
diffusion, which should be more pronounced in the former, can be traced. Identical in-plane structures are therefore essential.

A further peculiarity of the Li-GICs is the existence of two distinct stage- 2 phases: besides the ordered compound $\mathrm{LiC}_{12}$, which transforms above about $500 \mathrm{~K}$ to stage-1 with no long-range in-plane order [5], there exists a dilute compound, labeled $\mathrm{LiC}_{16}$, which has no long-range order already at room temperature $[5,6]$. The stage- 2 compounds thus offer the possibility to examine the role of in plane ordering in diffusion. Concerning the stage- 1 compound, the superstructure of $\mathrm{LiC}_{6}$ disappears at $715 \mathrm{~K}[7,8]$, thus exhibiting a disordered phase too, however in a restricted temperature range due to formation of lithium carbide above $750 \mathrm{~K}$.

The spectroscopic tools used for the investigations reviewed here are spin-lattice relaxation (SLR) and quasielastic neutron scattering (QENS). The former are performed with the method of $\beta$-radiation detected NMR ( $\beta$-NMR) with the probe nucleus ${ }^{8} \mathrm{Li}\left(T_{1 / 2}=0.8 \mathrm{~s}\right)$ on $\mathrm{LiC}_{6}$ and $\mathrm{LiC}_{12}$. For the stage- 2 compounds $\mathrm{LiC}_{12}$ and $\mathrm{LiC}_{16}$ neutron scattering data were obtained with time-of-flight (TOF) and backscattering (BS) spectrometers; data for the stage-1 compound $\mathrm{LiC}_{6}$ were taken from the literature for comparison. Both, neutron-scattering and $\beta$-NMR data, are used for the investigation of diffusive Li motion, and the combination of them allows to cover a wide range of jump frequencies. Furthermore the SLR results give access to electronic properties of the materials.

The paper is organised as follows. In the next chapter the samples, the spectrometers, and the techniques employed are briefly described. Then the results for each part, $\beta$-NMR and neutron scattering, are shown and discussed separately. In the last section the data concerning the diffusive behaviour of $\mathrm{Li}$ will be compiled and discussed.

\section{Experimental}

\subsection{Samples}

All samples were prepared from highly oriented pyrolytic graphite (HOPG) and isotopically pure ${ }^{7} \mathrm{Li}$ metal in order to avoid parasitic neutron absorption by ${ }^{6} \mathrm{Li}$ in both, the $\beta$-NMR and the neutron-scattering experiments. The samples were characterized by neutron diffraction for stage purity and $c$-axis mosaic spread. The stage- 2 admixture of the $\mathrm{LiC}_{6}$ samples was smaller than $3 \%$, for the stage- 2 samples a stage- 1 admixture below $10^{-4}$ was achieved. The $c$-axis mosaic spread was about $8^{\circ}$ full width at half maximum for all samples. The $\mathrm{LiC}_{12}$ samples used in the $\beta$-NMR investigation had a $15 \%$ admixture of the dilute stage- 2 phase $\mathrm{LiC}_{16}$. The stage-1 samples were prepared at the Materials Research Laboratory, University of Illinois at Urbana, the stage-2 samples were provided by the Laboratory for Research of the Structure of Matter, University of Pennsylvania at Philadelphia.

\section{$2.2 \beta$-Radiation Detected NMR}

The versatility of nuclear magnetic resonance/relaxation techniques for the investigation of both, structural and dynamic properties of intercalation compounds has been shown in, e.g., [9]. In this contribution $\beta$-radiation detected nuclear magnetic resonance/relaxation is used for the investigation of diffusion processes. Although comprehensive reviews on the $\beta$-NMR technique [10] and its application to the study of ion dynamics [11] are available, the method will briefly be described and its special features for the study of GICs will be discussed. In $\beta$-radiation detected NMR the $\beta$-radiation asymmetry of short-lived $\beta$-emitters in a sample is used to measure the nuclear polarization. Any changes of the nuclear polarization are observed via changes of the $\beta$-asymmetry.

The measurement proceeds in two steps: (i) generation of the short-lived radioactive nuclei in the sample and (ii) observation of the $\beta$-radiation asymmetry. Here for $(i)$ the capture of polarized thermal or cold neutrons by ${ }^{7} \mathrm{Li}$ nuclei in the sample is used. The observation of the $\beta$-radiation asymmetry is performed in a time-differential mode after neutron-activation pulses or under quasi-continuous activation condition by chopping the neutron beam fast compared to the $\beta$-lifetime. In the time-differential mode the decay behaviour of the nuclear polarization is observed directly, and the spin-lattice relaxation time $T_{1}$ can be obtained. Under quasi-continuous activation conditions resonance signals can be recorded.

Special features of the $\beta$-NMR technique for SLR measurements in GICs are the following. Since the nuclear polarization is created in a nuclear reaction, no Boltzmann factor is involved. Low values of the external $\boldsymbol{B}$ field are easily accessible. Further, the 
probe spins are extremely diluted and may be regarded as isolated spins with no spin temperature established. Mutual spin flips are suppressed, and the probes relax independently. Relaxation due to paramagnetic impurities does not contribute. Since the measurement of the SLR time does not require any radiofrequency irradiation, skin effect problems with the samples showing metallic conductivity do not arise. So metal single crystals can be used.

A limitation of the $\beta$-NMR method is the time window of accessible SLR times $T_{1}$ to the approximate range $0.1 T_{1 / 2} \leq T_{1} \leq 10 T_{1 / 2}$ around the nuclear half life $T_{1 / 2}$ of the probe nucleus. Furthermore, large samples of several $\mathrm{cm}^{3}$ are necessary in order to achieve sufficiently high count rates in the neutron activation experiment, a problem also encountered in the neutron scattering measurements.

The measurements were performed with the inbeam $\beta$-NMR spectrometer S6 $[10,11]$ at a thermal neutron guide of the high-flux reactor of the ILL Grenoble on $\mathrm{LiC}_{6}$, and with the $\beta$-NMR spectrometer at the DIDO reactor of the KFA Jülich [12] on the $\mathrm{LiC}_{12}$ sample. The cold neutron beam available at the latter compensates for the lower reactor power, yielding roughly the same measuring conditions.

\subsection{Quasielastic Neutron Scattering}

The neutron backscattering and the time-of-flight measurements on the stage- 2 compounds were performed with the IN10 and the IN6 spectrometers of the ILL. The scattering plane was always chosen perpendicular to the $c$-axis, thus probing in-plane diffusive motion. In both cases the momentum transfers $Q$ covered the range $Q=3 \mathrm{~nm}^{-1} \ldots 20 \mathrm{~nm}^{-1}$, and the energy resolution was about $1 \mu \mathrm{eV}$ for the BS and $70 \mu \mathrm{eV}$ for the TOF measurements.

In both, the BS and the TOF spectra, the quantity of interest here is the broadening of the elastic line of the incoherent scattering function $S_{\text {inc }}(Q, \omega)$ due to translational diffusion of the scatterer. With the usual approximations [13] the incoherent scattering functions reads

$$
S_{\mathrm{inc}}(\boldsymbol{Q}, \omega)=\frac{1}{\pi} \frac{\Gamma}{\Gamma^{2}+\omega^{2}} .
$$

For liquidlike diffusion of the scatterer with the diffusion constant $D$, the width $\Gamma$ is given by

$$
\Gamma=2 \hbar D Q^{2} \text {. }
$$

For jumplike particle motion the width depends on the mean residence time $\tau_{c}$ between jumps

$$
\Gamma=\frac{2 \hbar}{\tau_{c}} f(Q),
$$

where $f(\boldsymbol{Q})$ is a model dependent geometric function given by

$$
f(Q)=\frac{1}{n} \sum_{i=1}^{n}\left[1-\exp \left(-i \boldsymbol{Q} \cdot \boldsymbol{l}_{i}\right)\right] .
$$

The sum is taken over all possible neighbour sites of the particle accessible with a jump vector $\boldsymbol{l}_{i}$. Thus inspection of $\Gamma(Q)$ offers, unlike the SLR measurements, direct access to the geometry of the diffusion process.

\section{Results and Discussion}

\subsection{Spin-Lattice Relaxation of ${ }^{8} \mathrm{Li}$}

\subsubsection{Overview}

For the stage- 2 compound $\mathrm{LiC}_{12}$ the transients of the $\beta$-radiation asymmetry showed single-exponential decay behaviour in the entire range of temperatures and of $B$-field values for both crystal orientations $\boldsymbol{B} \| \boldsymbol{C}$ and $\boldsymbol{B} \perp \boldsymbol{c}$. For $\mathrm{LiC}_{6}$ the same observation was made except in a $T$ range below $300 \mathrm{~K}$, which will be discussed below. Figure 1 gives an overview of $T_{1}^{-1}(T)$ of ${ }^{8} \mathrm{Li}$ in $\mathrm{LiC}_{12}$ for the two crystal orientations and a single $B$ value. Four regions of different $T$-dependences may be distinguished: (i) For $T<100 \mathrm{~K}_{1}^{-1}$ shows a linear $T$ dependence and is independent of $B$ and the crystal orientation. The high-temperature extrapolation of this SLR process is indicated by the dotted line in Figure 1. (ii) For $100 \mathrm{~K}<T<300 \mathrm{~K} T_{1}^{-1}$ is still independent of the crystal orientation and exceeds the linear $T$ dependence extrapolated from lower temperatures. (iii) For $300 \mathrm{~K}<T<500 \mathrm{~K}_{1}^{-1}$ shows a steep increase and a maximum induced by $\mathrm{Li}$ diffusion. The rate depends on the value of $B$ as well as the crystal orientation, as illustrated in Figure 2. (iv) for $T>500 \mathrm{~K} T_{1}^{-1}$ does not reduce to the high- $T$ extrapolation of the SLR process observed in the low- $T$ region. Thus a further $T_{1}^{-1}$ contribution becomes dominant above about $500 \mathrm{~K}$. For the stage- 1 compound a similar diffusion induced rate maximum between about $300 \mathrm{~K}$ and $500 \mathrm{~K}$, induced by Li diffusion, and an additional SLR process arising above 


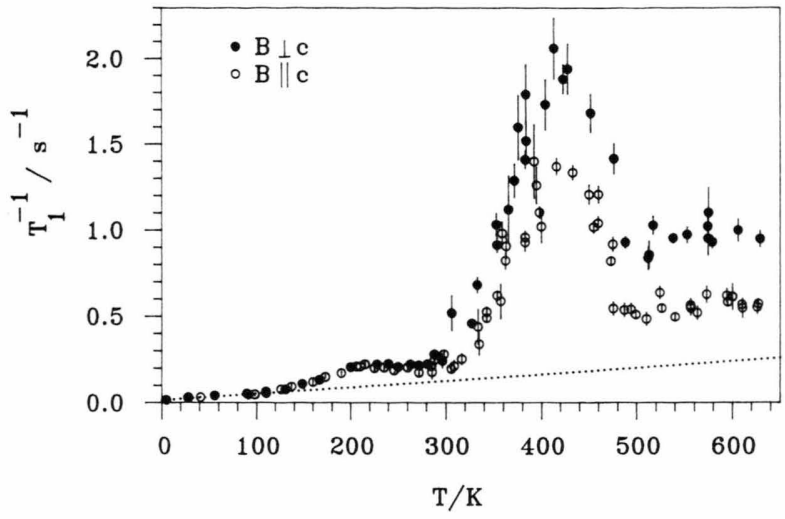

Fig. 1. $T$ dependence of $T_{1}^{-1}$ of ${ }^{8} \mathrm{Li}$ in $\mathrm{LiC}_{12}$ for $B=37 \mathrm{mT}$ and two crystal orientations. The dotted line marks the SLR contribution due to coupling to conduction electrons showing up as a background at higher temperatures.

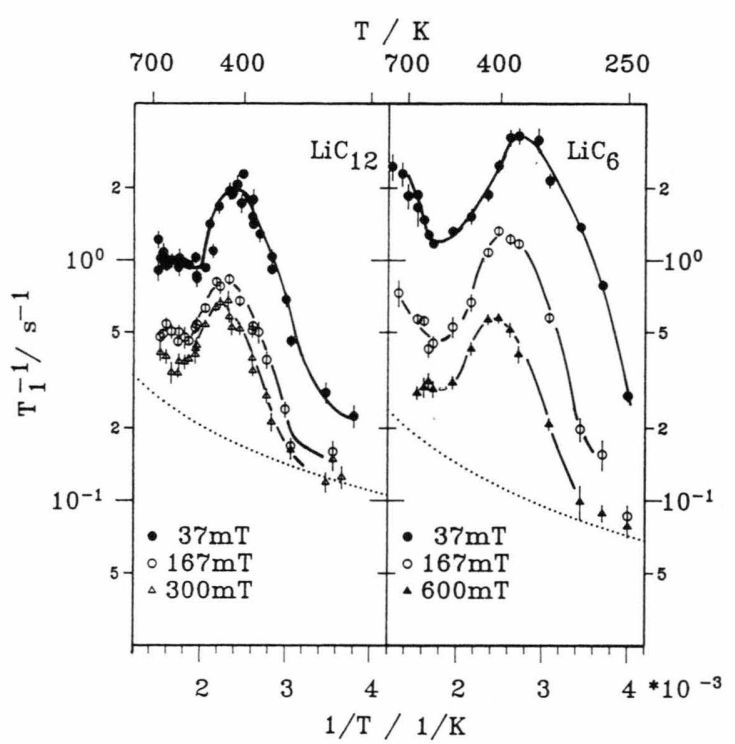

Fig. 2. SLR rate $T_{1}^{-1}$ of ${ }^{8} \mathrm{Li}$ in $\mathrm{LiC}_{12}$ (left) and $\mathrm{LiC}_{6}$ (right) at various $B$ for $\boldsymbol{B} \perp \boldsymbol{c}$. The solid lines are a guide to the eye, the dotted line for $\mathrm{LiC}_{12}$ is the high- $T$ extrapolation of the electronic SLR contribution observed below $100 \mathrm{~K}$. The dotted line for $\mathrm{LiC}_{6}$ is the background rate according to $T_{1 \mathrm{~b}} T=3500 \mathrm{sK}$.

$500 \mathrm{~K}$ similar to the $T_{1}^{-1}$ behaviour in (iv) showed up [14]. The discussion will mainly be concerned with the long-range $\mathrm{Li}$ diffusion dominating $T_{1}^{-1}$ in region (iii).

\subsubsection{Spin-Lattice Relaxation Below $300 \mathrm{~K}$}

Since SLR processes being dominant in the $T$ ranges ( $i$ ) and (ii) might also contribute in the $T$ range (iii) of the pronounced peak, a short description with emphasis on $\mathrm{LiC}_{12}$, which was studied more extensively, will be given here. A more detailed discussion is presented in [15].

The linearly $T$ dependent SLR contribution below $100 \mathrm{~K}$ is due to coupling of the ${ }^{8} \mathrm{Li}$ probe spins to conduction electrons. For this contribution the Korringa relation

$$
T_{1} T K^{2} \eta=\frac{\hbar}{4 \pi k_{B}}\left(\frac{\gamma_{\mathrm{e}}}{\gamma_{\mathrm{n}}}\right)^{2}=S_{0}
$$

holds, where $\gamma_{\mathrm{e}}$ and $\gamma_{\mathrm{n}}$ are the gyromagnetic ratio of the electron and the probe nucleus, respectively. $K$ denotes the Knight shift. The Korringa-enhancement factor $\eta$ accounts for deviations of the system from free-electron behaviour. Values $\eta<1$ usually found in metals are due to electron-electron interaction, values $\eta>1$ may be ascribed to ion-electron interaction, and values $\eta \gg 1$ are found in low-D electronic systems [16]. Combination of the $T_{1} T$ value of ${ }^{8} \mathrm{Li}$ with the Knight shift of ${ }^{7} \mathrm{Li}$ obtained at room temperature for the same $\mathrm{LiC}_{12}$ sample [17] yields $\eta \simeq 3.5$. The earlier estimate $\eta \simeq 2$ [15] stems from a higher Knight shift value of a powder sample given in the literature [18] and might be an underestimate. The spread of the $\eta$ values does, however, not affect the conclusion of a low-D electronic character of $\mathrm{LiC}_{12}$.

For the stage-1 compound $\mathrm{LiC}_{6}$, SLR data for $T$ below $100 \mathrm{~K}$ are too scarce for a detailed comparison. In the high- $T$ region the SLR contribution due to coupling to conduction electrons does contribute to the experimental $T_{1}^{-1}$ value in $\mathrm{LiC}_{6}$ as a background rate $T_{1 \mathrm{~b}}^{-1}$ in a similar way as illustrated in Figure 1. In $\mathrm{LiC}_{6}$ the temperature dependence of $T_{1 \mathrm{~b}}^{-1}$ was estimated as $T_{1 \mathrm{~b}} T \approx 3500 \mathrm{sK}$ [14], in $\mathrm{LiC}_{12}$ the $T$ dependent part of the background contribution is described by $T_{1 \mathrm{~b}} T \approx 2600 \mathrm{sK}$.

Between $100 \mathrm{~K}$ and $300 \mathrm{~K}$ SLR contribution in addition to the conduction-electron induced SLR shows up in $\mathrm{LiC}_{12}$ as illustrated in Figure 1. The semilogarithmic plot of this additional rate vs. $1 / T$ exhibits a linear increase above $100 \mathrm{~K}$ and a maximum near $200 \mathrm{~K}$, which both are characteristic of motion induced SLR. The slope on the low- $T$ side of this secondary peak corresponds to an activation energy of about $50 \mathrm{meV}$. The additional SLR contribution shows a weak $B$ dependence which can be approximated by a power law $B^{-0.5}$. This is weaker than the $B^{-2}$ dependence predicted by the standard model for motion induced SLR by Bloembergen et al. (BPP) [19]. 
Consequently, the value given for the activation energy is only a lower estimate. It should be noted that similar weak $B$ dependences of the SLR rate were found with ${ }^{7} \mathrm{Li} \mathrm{NMR}$ in crystalline $\beta$ - $\mathrm{LiAlSi}_{2} \mathrm{O}_{6}$, which is a Li-ion conductor too [20].

In $\mathrm{LiC}_{6}$ a nonexponential behaviour of the $\beta$-asymmetry transients of ${ }^{8} \mathrm{Li}$ for $T$ between $100 \mathrm{~K}$ and $270 \mathrm{~K}$ was observed which can be described by a double exponential decay law with time constants $T_{1}^{(1)}$ and $T_{1}^{(2)}\left(T_{1}^{(1)}<T_{1}^{(2)}\right)$. The $B$ dependence of both, $1 / T_{1}^{(1)}$ and $1 / T_{1}^{(2)}$, is weaker than $\propto B^{-2}$. The $T$ dependence of the SLR rate corresponding to the shorter decay time constant $T_{1}^{(1)}$ exhibits a peak. From the slope of the low- $T$ side of the peak, in the $\log \left(1 / T_{1}^{(1)}\right)$ vs. $1 / T$ representation an activation energy of $75 \mathrm{meV}$ is estimated, which is as in the case of $\mathrm{LiC}_{12}$ only a lower limit.

The mechanisms, whether dipolar or quadrupolar, and consequently the origin of the SLR processes arising additionally to the electron contribution in both, $\mathrm{LiC}_{6}$ and $\mathrm{LiC}_{12}$, are not clear. A comparison with ${ }^{7} \mathrm{Li}$ NMR data for $\mathrm{LiC}_{6}[21]$ is hampered by the different $B$ dependences found. Lauginie et al. [21] report a $T_{1}^{-1}$ value of ${ }^{6} \mathrm{Li}$ at $200 \mathrm{~K}$ which is consistent with the conduction-electron induced SLR contribution of ${ }^{8} \mathrm{Li}$. Due to the small quadrupole moment of ${ }^{6} \mathrm{Li}$ this is a hint that the additional SLR process observed for ${ }^{8} \mathrm{Li}$ in $\mathrm{LiC}_{6}$ is quadrupolar. It might be related to a suggested phase transition at about $200 \mathrm{~K}$ ([22] and references therein). In the $\mathrm{LiC}_{12}$ sample the SLR behaviour might be influenced by the known phase transition at $T=248 \mathrm{~K}$ [23] in the minority $\mathrm{LiC}_{16}$ phase. The relatively low abundance of the latter, together with a possible translational $\mathrm{Li}$ motion during the lifetime of the ${ }^{8} \mathrm{Li}$ probe might effect an averaging over inequivalent probe surroundings and prevent a nonexponential decay of the $\beta$-asymmetry of ${ }^{8} \mathrm{Li}$ in the stage- 2 sample.

\subsubsection{Spin-Lattice Relaxation Above $300 \mathrm{~K}$}

In both, $\mathrm{LiC}_{6}$ and $\mathrm{LiC}_{12}$, the SLR rate increases drastically with $T$ above $300 \mathrm{~K}$ and shows a diffusioninduced rate maximum as illustrated in Fig. 2 in the usual $\log \left(T_{1}^{-1}\right)$ vs. $1 / T$ representation for the orientation $\boldsymbol{B} \perp \boldsymbol{c}$. For the orientation $\boldsymbol{B} \| \boldsymbol{c}$ similar $T_{1}^{-1}(T)$ peaks are observed. In both compounds the SLR due to coupling to conduction electrons contributes to the observed SLR rate in the $T$ range of the diffusion induced rate maximum and is treated as a background

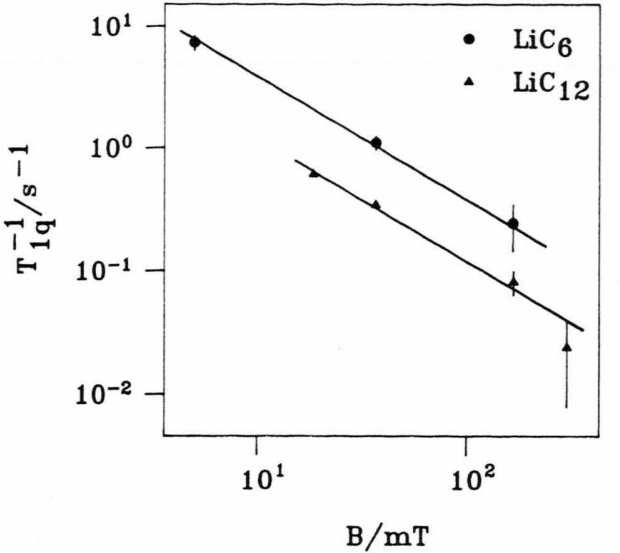

Fig. 3. The SLR contribution $T_{1 \mathrm{a}}^{-1}$ to the SLR rate of ${ }^{8} \mathrm{Li}$ in $\mathrm{LiC}_{6}(T=600 \mathrm{~K})$ and $\mathrm{LiC}_{12}(T=595 \mathrm{~K})$ as a function of the $\boldsymbol{B}$ field for $\boldsymbol{B} \perp \boldsymbol{c}$ in double-logarithmic representation. The lines represent a $B^{-1}$ dependence.

rate $T_{1 \mathrm{~b}}^{-1}$. It is given by the dotted lines in Fig. 2 and has to be subtracted from the experimental $T_{1}^{-1}$ values in order to obtain the diffusion-induced rate $T_{1 \mathrm{~d}}^{-1}$ which will be discussed further.

In the $T$ range (iv) above the high- $T$ side of the rate peaks, $T_{1}^{-1}$ does not decrease to $T_{1 \mathrm{~b}}^{-1}$ for $\mathrm{LiC}_{6}$ and $\mathrm{LiC}_{12}$. A further SLR contribution denoted as $T_{1 \mathrm{q}}^{-1}$ shows up. It is larger in the stage-1 compound and anisotropic with $\left(T_{1 \mathrm{q}}^{-1}\right)_{B \perp c}>\left(T_{1 \mathrm{q}}^{-1}\right)_{\boldsymbol{B} \| \boldsymbol{c}}$. The anisotropy is larger for $\mathrm{LiC}_{6} . T_{1 \mathrm{q}}^{-1}$ is $B$ dependent as illustrated by Figure 3. The $B$ dependence follows a $T_{1 \mathrm{q}}^{-1} \propto B^{-1}$ law in $\mathrm{LiC}_{6}$ and $\mathrm{LiC}_{12}$. The large anisotropy suggests quadrupolar coupling $[24,25]$. The origin of a quadrupolar SLR mechanism is, however, not entirely clear. At the elevated temperatures both compounds contain vacancies in high concentration, and multiplevacancy diffusion with correlation times larger than those of the low- $T$ phase might be possible.

The high-T SLR behaviour in the Li GICs is qualitatively similar to that in $\mathrm{Li}$ intercalated hexagonal $\mathrm{TiS}_{2}$ investigated with ${ }^{7} \mathrm{Li}-\mathrm{NMR}$ [26]. A diffusion induced rate maximum and an additional high- $T$ SLR process were observed there, too.

Returning to the diffusion induced rate maxima in $\mathrm{LiC}_{6}$ and $\mathrm{LiC}_{12}$, which have been described singly in [14] and [27], the discussion here will concentrate on the comparison of the compounds. For both, the $B$ dependence of the SLR rate showed deviations from the behaviour predicted by standard models for motion induced SLR. On the low- $T$ side of the peak and at the peaks themselves the $B$ dependences were 
weaker. On the the high- $T$ side a dispersion of the SLR rate with $B$ was found. Its inspection turned out to be indispensable to elucidate the role of low-D diffusion.

\subsubsection{Diffusion Induced Spin-Lattice Relaxation}

The evaluation of diffusion parameters from SLR data is achieved by invoking spectral density functions $J(\omega)$ which are the Fourier transforms of the correlation functions $G(\tau)$ describing the motional process. The diffusion induced SLR of ${ }^{8} \mathrm{Li}$ in the temperature range (iii) is caused by fluctuations of the dipoledipole interaction. Evidence for the relaxation mechanism being dipolar comes from the $B$ dependence on the high- $T$ side of the rate maximum, which is a feature of low-D diffusion. For the case that the diffusion jump rate $\tau_{c}^{-1}$ is large in comparison with the Larmor frequency $\omega_{0}\left(\omega_{0} \tau_{c} \ll 1\right)$, which is fulfilled on the high$T$ side, only in the expression for dipolar SLR spectral density functions with an $\omega$ dependence do occur $[14,25]$. Further support for a dipolar mechanism comes from a calculation of the lattice sums which determine the peak $T_{1 \mathrm{~d}}^{-1}$ value for dipolar coupling [28]. For both, the ratio of the $\left(T_{1 \mathrm{~d}}^{-1}\right)_{\max }$ values of $\mathrm{LiC}_{6}$ and $\mathrm{LiC}_{12}$ and the anisotropy of $\left(T_{1 \mathrm{~d}}^{-1}\right)_{\max }$ for $\boldsymbol{B} \| \boldsymbol{c}$ and $\boldsymbol{B} \perp \boldsymbol{c}$, the calculations agree with the experimental values.

The usual way to obtain diffusion parameters from the $T_{1 \mathrm{~d}}^{-1}$ data by fitting spectral density functions $J(\omega)$ from standard models is hampered by the weak $\omega_{0}(\triangleq B)$ dependence on the low- $T$ side of the rate maximum. Here $\omega_{0} \tau_{c} \gg 1$ holds, and the short time behaviour $\left(\tau \ll \tau_{c}\right)$ of $G(\tau)$ is probed. The deviation of $G(\tau)$ from single exponential decay behaviour, which is reflected by the $\omega$ dependence being weaker than $\omega^{-2}$, can be characterized by a constant $0<\alpha<1$ in $G\left(\tau \ll \tau_{c}\right) \propto \exp \left[-\left(\tau / \tau_{c}\right)^{1-\alpha}\right][29,30]$. This is equivalent to a modified spectral density function

$$
J(\omega) \propto \tau_{c}\left(\omega \tau_{c}\right)^{-2+\alpha} .
$$

The temperature dependence of the characteristic time for motional processes is given by

$$
\tau_{c}=\tau_{c 0} \exp \left[E_{\mathrm{A}} / k T\right]
$$

where $E_{\mathrm{A}}$ is the activation energy. With $\alpha$ determined by the $\omega$ dependence, $E_{\mathrm{A}}$ values can be estimated. The low- $T$ side of the $\log \left(T_{1 \mathrm{~d}}^{-1}\right)$ vs. $1 / T$ plots yields $0.6(2) \mathrm{eV}$ for $\mathrm{LiC}_{6}$ and $1.0(3) \mathrm{eV}$ for $\mathrm{LiC}_{12}$.
These estimates are supported by the inspection of the SLR-rate maxima. The temperature $T_{\max }$ where $T_{1 \mathrm{~d}}^{-1}$ attains its maximum shifts with rising Larmor frequencies to higher temperatures. This shift may be used for another estimate of the activation energy. Regardless of the microscopic details of the diffusion jump mechanism, the spectral density function $J(\omega)$ attains its maximum for $\omega_{0} \simeq \tau_{c}^{-1}$. The shift of $T_{\max }$ yields $E_{\mathrm{A}} \approx 0.7 \mathrm{eV}$ for $\mathrm{LiC}_{6}$ and $E_{\mathrm{A}} \approx 0.9 \mathrm{eV}$ for $\mathrm{LiC}_{12}$. The $\tau_{c}$ values obtained in this way are discussed in Section 3.3. Contrary to $E_{\mathrm{A}}$ the estimate of $\tau_{c}$ values is subjected to a common systematic error, since the exact value of $\omega_{0} \tau_{c}$ at the rate maximum is not known in the present case of non-BPP behaviour.

The observed nonexponential correlation functions are a common feature of superionic conductors [30]. In computer simulations [31] the important role of structural disorder and Coulomb interactions for the deviation of the SLR behaviour from the standard model for diffusion induced SLR was shown. However, in the present case the origin of the observed nonexponential correlation functions is not clear. Inspection of the phonon density of states of $\mathrm{LiC}_{6}$ reveals a strong screening of the Coulomb interaction in comparison with heavy alkali GICs [32], where the Coulombic-force model without screening was more appropriate for the description of the phonon density of states. Further, the compounds investigated here should be well ordered in the discussed $T$ range with the admixture of disordered phases being low. In the frame of the Daumas-Hérold model of small ordered domains [33], disorder effects on diffusion induced SLR might, however, be conceived in case that the diffusion length within a time of the order of $T_{1 \mathrm{~d}}$ exceeds the supposed size of $2 \mathrm{D}$ intercalate "islands".

At temperatures above the rate maximum the condition $\omega_{0} \tau_{c} \ll 1$ holds and the long-time behaviour of $G\left(\tau \gg \tau_{c}\right)$ is probed. The standard model predicts for the spectral density $J \propto \tau_{c}$ with $J$ independent of $\omega$, which is at variance with the data. Figure 4 shows examples of the $B$ dependence observed in $\mathrm{LiC}_{6}$ and $\mathrm{LiC}_{12}$ on the high- $T$ side of the maximum.

Theoretical calculations for a $2 \mathrm{D}$ diffusion process yield for the case $\omega \tau_{c} \ll 1$ the expression [24]

$$
J\left(\omega \ll \tau_{c}^{-1}\right) \propto \tau_{c} \ln \frac{1}{\omega \tau_{c}} .
$$

Figure 4 is a plot of $T_{1 \mathrm{~d}}^{-1}(B)$ with a logarithmic $B$ scale. The data for both compounds are in accordance with a $2 \mathrm{D}$ process. It should be noted that the calculation 


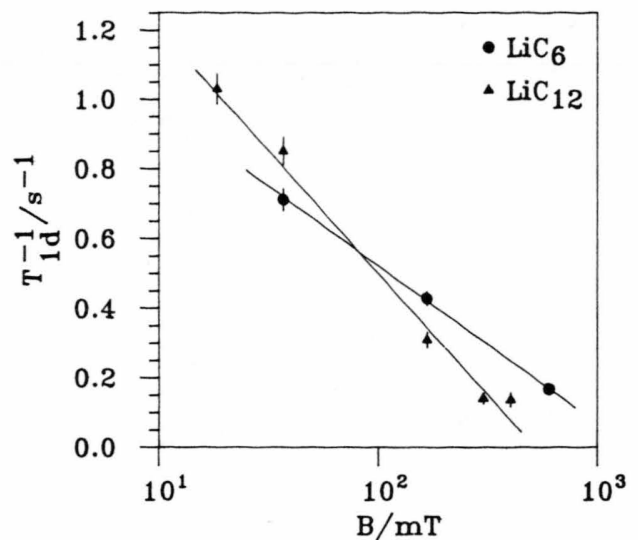

Fig. 4. $T_{1 \mathrm{~d}}^{-1}$ of ${ }^{8} \mathrm{Li}$ in $\mathrm{LiC}_{6}(T=462 \mathrm{~K}, \boldsymbol{B} \| c)$ and $\mathrm{LiC}_{12}$ $(T=460 \mathrm{~K}, \boldsymbol{B} \| c)$ as a function of the $B$ field (on logarithmic scale) at temperatures on the high- $T$ side of the rate maximum. The solid lines are fits with (8).

of $T_{1 \mathrm{~d}}^{-1}$ from the experimental $T_{1}^{-1}$ values involves the subtraction of the SLR contribution due to coupling to electrons denoted as $T_{1 \mathrm{~b}}^{-1}$ as well as a contribution from the high- $T$ SLR process $T_{1 \mathrm{q}}^{-1}$ at the respective temperature and $B$ value. The procedure is subjected to the uncertainty of this extrapolation, since the origin of $T_{1 \mathrm{q}}^{-1}$ is unclear. Without this latter correction, intriguingly the data for $\mathrm{LiC}_{6}$ turned out to be compatible with the $\omega^{-1 / 2}$ law predicted for $1 \mathrm{D}$ diffusion $[14,22]$. As things are, however, the SLR results for $\mathrm{LiC}_{6}$ as well as for $\mathrm{LiC}_{12}$ strongly support the conjecture of $2 \mathrm{D}$ diffusion of the $\mathrm{Li}$ ions in their planes between graphite layers, and the logarithmic $B$ dependence of $T_{1 \mathrm{~d}}^{-1}$ was used to obtain values for $\tau_{c}$ according to (8). These will be discussed in Section 3.3.

\subsection{Quasielastic Line Broadening of $S_{\mathrm{inc}}(Q, \omega)$}

In this section neutron time-of-flight and backscattering measurements on the stage- 2 Li-GICs $\mathrm{LiC}_{12}$ and $\mathrm{LiC}_{16}$ will be used for the study of the diffusional $\mathrm{Li}$ in-plane motion. For comparison with the stage-1 compound comprehensive reviews on neutron scattering investigations on $\mathrm{LiC}_{6}$ are available $[1,13]$.

In backscattering measurements on $\mathrm{LiC}_{12}$ no broadening of the quasielastic line was observed but a decrease of the elastic intensity $I$ integrated over the instrumental resolution as the temperature was raised from $T=50 \mathrm{~K}$ to $T>600 \mathrm{~K}$ [34]. The elastic intensity in these fixed-window scans decreases due to the re- duction of the Debye-Waller factor and the onset of motional processes in the frequency window of the instrumental resolution. In the fixed-window scans of $\mathrm{LiC}_{12}$ and $\mathrm{LiC}_{16}$ the former results in a weak decrease with $T$ already at low temperatures. Reduction of $I$ due to diffusion is more pronounced and occurs above about $300 \mathrm{~K}$. Thus for $\mathrm{LiC}_{12}$ the fixed-window scans give access only to the diffusion process observed by SLR above $300 \mathrm{~K}$. Motional processes between $100 \mathrm{~K}$ and $300 \mathrm{~K}$, being discussed in Subsection 3.1.2, are not reflected in decreases of $I$ in the neutron spectra at the same temperatures. Considering the low frequencies of the $\beta$-NMR measurements (in the region of $10^{6} \mathrm{~Hz}$ ) and the low activation energies estimated, a signal in the fixed-window measurements (in the $10^{9} \mathrm{~Hz}$ region) is expected above $350 \mathrm{~K}$, i.e. in the $T$ range where long-range $\mathrm{Li}$ diffusion becomes effective.

For the compound $\mathrm{LiC}_{16}$, having no long-range $\mathrm{Li}$ order, a quasielastic line broadening showed up in the backscattering spectra. The elastic intensity is evaluated with the usual procedure of convoluting a Lorentzian and a $\delta$ function with the instrumental resolution function. The linewidth $\Gamma(Q)$ thus obtained is plotted in the upper part of Figure 5. For the further treatment of $\Gamma(Q)$ in terms of an in-plane jump diffu-

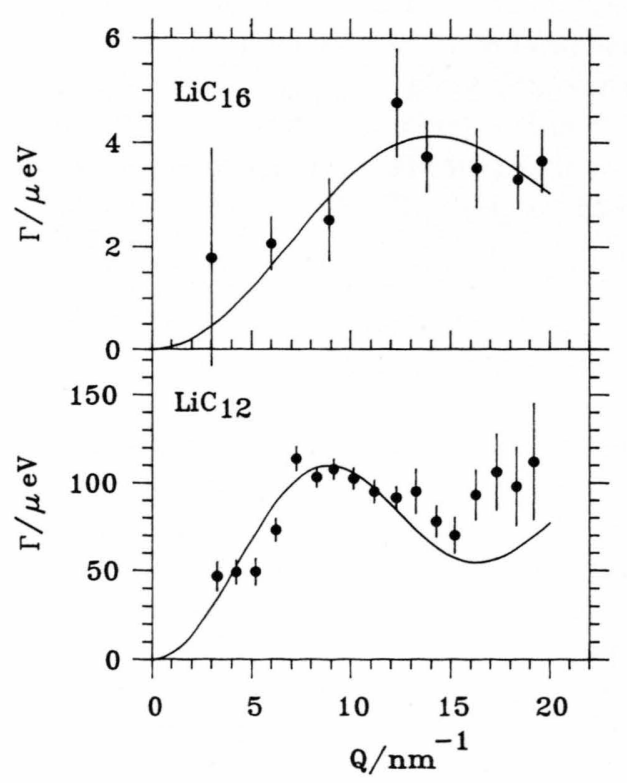

Fig. 5. Linewidths of the quasielastic neutron backscattering and time-of-flight spectra for $\mathrm{LiC}_{16}(T=450 \mathrm{~K})$ and $\mathrm{LiC}_{12}$ $(T=650 \mathrm{~K})$ as functions of the wave vector $Q$. The solid lines are fits with (9). 
sion process according to (4), a proper $2 \mathrm{D}$ average has to be performed in order to account for the orientational randomness of the $a$ or $b$ axes of the crystallites of the HOPG. It yields

$$
\Gamma(Q)=\frac{2 \hbar}{\tau_{c}}\left(1-J_{0}(Q l)\right),
$$

where $J_{0}$ is the zeroth order Bessel function; $\tau_{c}$ and $l$ are the jump correlation time and the jump length, respectively. The solid line through the data for $\mathrm{LiC}_{16}$ in Fig. 5 is a fit with (9) yielding $\tau_{c}$ and $l$.

The observation of a diffusion induced reduction of the fixed window intensity but of no quasielastic broadening in the case of $\mathrm{LiC}_{12}$ is due to the narrow dynamic range of the backscattering spectrometer. This was circumvented by measuring with a time-offlight spectrometer with a poorer energy resolution but a larger energy range. Quasielastic line broadening data for $T=650 \mathrm{~K}$ are shown in the lower part of Figure 5. Only for this $T$ a maximum in $\Gamma(Q)$ was observed, allowing the determination of $\tau_{c}$ as well as $l$. At lower $T$, maxima in $\Gamma(Q)$ could not be resolved due to the limited statistic accuracy. A $\Gamma \propto Q^{2}$ behaviour for the low $Q$ range in accordance with (2) was observed and used for the evaluation of diffusion coefficients (see Section 3.3). For $\mathrm{LiC}_{6}$ and $\mathrm{LiC}_{12}$ the measured values for $l$ are close to the distance $0.426 \mathrm{~nm}$ of next-nearest neighbour sites in the $(\sqrt{3} \times \sqrt{3}) R 30^{\circ}$ superstructure [35]. For $\mathrm{LiC}_{16} l$ close to the in-plane distance $0.25 \mathrm{~nm}$ of hexagon centres, i.e. the distance from a Li site to the site in the centre between adjacent carbon hexagons [34].

\subsection{Diffusivity in Stage-1 and Stage-2}

In this section, diffusion parameters obtained by the $\beta$-NMR and the neutron scattering techniques are summarized. The diffusion coefficients $D$ were obtained directly by fitting (2) to the quasielastic linewidth data when no maximum in $\Gamma(Q)$ was resolved. For the $\mathrm{LiC}_{16}$ data and the highest temperature run for $\mathrm{LiC}_{12}$, (9) was fitted to the $\Gamma(Q)$ values, and the diffusion coefficient was calculated from $\tau_{c}$ and $l$ according to the expression for $2 \mathrm{D}$ diffusion

$$
D=\frac{l^{2}}{4 \tau_{c}} .
$$

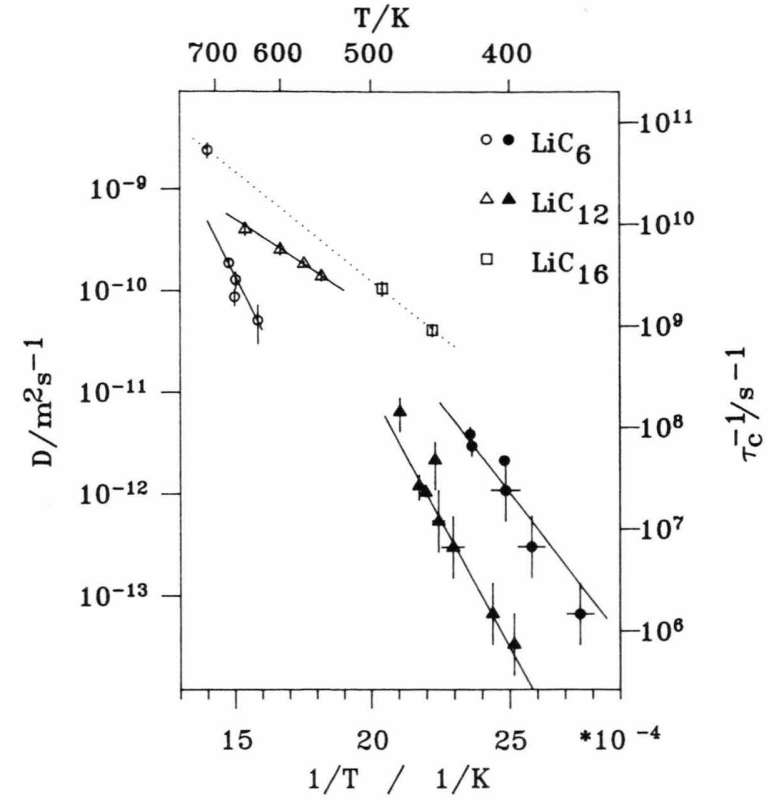

Fig. 6. Synoptic representation of the temperature dependence of the diffusion coefficient (left-hand ordinate) and jump rate (right-hand ordinate) for the in-plane Li diffusion in various Li-GICs obtained with quasielastic neutron scattering (open symbols) and $\beta$-NMR (full symbols). Horizontal error bars refer to the uncertainty of the temperature determination were SLR rate maxima occur, vertical error bars account for the uncertainty of the value of $\omega_{0} \tau_{c}$ at the rate maxima. The QENS data for $\mathrm{LiC}_{6}$ were taken from [35]. The solid lines correspond to activation energies given in the text. The dotted line corresponds to a common activation energy for $\mathrm{LiC}_{16}$ and the disordered phase of $\mathrm{LiC}_{6}$.

The jump length in $\mathrm{LiC}_{12}$ is $l=0.48(2) \mathrm{nm}$. For $\mathrm{LiC}_{6}$ a compatible value for $l$ was observed [35], for $\mathrm{LiC}_{16}$ the mean value $l=0.276(14) \mathrm{nm}$ was found.

The diffusion coefficients are shown in Fig. 6 together with the jump-correlation rates $\tau_{c}^{-1}$ for $\mathrm{LiC}_{6}$ and $\mathrm{LiC}_{12}$ obtained by the $\beta$-NMR measurements. The shift of the scales for $D$ and $\tau_{c}^{-1}$ corresponds to an in-plane jump-distance equal to the next-nearest hexagon centres in (10). This implies that the same 2 D in-plane diffusion mechanism are seen in the QENS and $\beta$-NMR measurements. For $\mathrm{LiC}_{6}$ this scaling results in a discontinuity of the extrapolation from the $D$ values obtained by QENS to the $\tau_{c}^{-1}$ values obtained by $\beta$-NMR.

In $\mathrm{LiC}_{6}$ the quasielastic line broadening yielded an activation energy of $1.0(3) \mathrm{eV}$ [35], the $\beta$-NMR data the slightly lower $E_{\mathrm{A}}$ value $0.6(2) \mathrm{eV}$ [14]. In both techniques the $T$ range of the well ordered phase of $\mathrm{LiC}_{6}$ was covered. Differences in the extracted $E_{\mathrm{A}}$ values 
might arise from the different time windows of the spectroscopic methods, which are about three orders of magnitude apart. The $\beta$-NMR data showed pronounced deviations from Lorentzian shaped spectral density functions. The evaluation of the quasielastic line broadening of the neutron spectra was, due to the statistics, limited to Lorentzian scattering functions. The $D$ value at $720 \mathrm{~K}$ pertains to the disordered state above the in-plane melting temperature of $715 \mathrm{~K}[7,8]$ and shows a dramatic increase of the diffusivity. This illustrates the influence of structural changes on $E_{\mathrm{A}}$.

For $\mathrm{LiC}_{12}$ the TOF spectra yield an activation energy $E_{\mathrm{A}}=0.35 \mathrm{eV}$ [34]. This is considerably lower than the $E_{\mathrm{A}}$ value of about $1 \mathrm{eV}$ estimated from the $\beta$-NMR data. It is noted that the $\beta$-NMR data cover temperatures below the order/disorder transition at about $500 \mathrm{~K}$, while the TOF data cover the disordered phase of $\mathrm{LiC}_{12}$. In Fig. 6 the turn-over of the slope of the $\tau_{c}^{-1}(T)$ data to that of the $D(T)$ data is located close to the transition temperature. So different jump motions below and above the transition might lead to the difference in $E_{\mathrm{A}}$. It was already mentioned in the previous section that the QENS spectra at the lower temperatures were not analysed in terms of jump vectors and correlation times, and a change in the jump geometry cannot be traced further within the accuracy of the present data.

For $\mathrm{LiC}_{16}$ the data are too scarce for a reliable determination of $E_{\mathrm{A}}$, and only an estimate can be given. The jump vector agrees with that found in the disordered phase of $\mathrm{LiC}_{6}$. It should be noted that for $\mathrm{LiC}_{6}$ and $\mathrm{LiC}_{16}$ in their respective disordered states the diffusivities may be described by a common $E_{\mathrm{A}}$ value for which $0.43 \mathrm{eV}$ is estimated. This is close to the value found for the disordered phase of $\mathrm{LiC}_{12}$ and is a hint that interplanar couplings play a minor role for the ionic motion in the disordered phase and that the activation energy in the stage- 1 compound is reduced upon becoming disordered as supposed earlier [13]. A similar observation was made in diffusion studies in hexagonal $\mathrm{Li}_{x} \mathrm{TiS}_{2}$. There, upon depletion of the Li layers a reduction of the activation energy was observed [26].

Lattice simulation calculations of the $\mathrm{Li}$ in-plane migration for $\mathrm{LiC}_{6}$ and $\mathrm{LiC}_{12}$ reveal the same jump vector as observed in the neutron scattering experiments [36]. The saddle point energy for the migration path is in close agreement with the experimental result for $\mathrm{LiC}_{12}$. But the simulations yield a lower saddle point energy for $\mathrm{LiC}_{6}$, a finding corresponding to the
$\beta$-NMR data. Recent Monte Carlo simulations of the Li diffusion through a graphite lattice based on cluster calculations of the electronic structure also yield energy barriers for the $\mathrm{Li}^{+}$migration [37]. For the inplane diffusion of a single $\mathrm{Li}$ atom an activation energy of $0.8 \mathrm{eV}$ is obtained, for the preexponential $D_{0}$ the value $10^{-6} \mathrm{~m}^{2} \mathrm{~s}^{-1}$ is reported. This is of the order of magnitude found for $\mathrm{LiC}_{12}$. The cluster calculations yield an energy barrier of $13 \mathrm{eV}$ for the diffusion path along the $c$ direction, ruling out motions across the graphene sheets in the intercalation process. The simulations further emphasize the crucial role of the Coulomb interaction of the $\mathrm{Li}^{+}$diffusion.

\subsection{Summary}

Motional processes in stage-1 and stage- 2 Li graphite intercalation compounds were investigated in a wide range of temperatures with the $\beta$-NMR method and additionally with neutron time-of-flight and backscattering techniques in the high temperature regime.

Below $100 \mathrm{~K}$ coupling to conduction electrons dominates the SLR. For $\mathrm{LiC}_{12}, T_{1}^{-1}$ and Knight shift data were used to calculate the Korringa product. An ionic character of $\mathrm{Li}$ and low-dimensional electronic properties of the compound in accordance with theoretical considerations were found.

Above $300 \mathrm{~K}$, diffusion induced SLR peaks were observed in $\mathrm{LiC}_{6}$ and $\mathrm{LiC}_{12}$. The $B$ dependence on the low- $T$ side of the maximum is weaker than predicted by standard models for diffusion induced SLR and was used to estimate activation energies from the slope of the $\log \left(T_{1 \mathrm{~d}}^{-1}\right)$ vs. $1 / T$ representation. The $B$ dependence on the high- $T$ side of the rate maximum is compatible with a $2 \mathrm{D}$ diffusion process. From the shift of the temperature where the rate maximum is attained with $B$ as well as from the $B$ dependence on the high- $T$ side jump correlation times $\tau_{c}$ were estimated. The neutron scattering experiments on the stage- 2 compounds were restricted to the high- $T$ regime and yielded in-plane diffusion constants and jump vectors.

The activation energy obtained by $\beta$-NMR for $\mathrm{LiC}_{6}$ is $0.6 \mathrm{eV}$. This is slightly below the literature value obtained by QENS. The difference may be attributed to the different frequency windows of the methods. For $\mathrm{LiC}_{12}$ TOF measurements yielded $E_{\mathrm{A}}=0.35 \mathrm{eV}$, the $\beta$-NMR data about $1 \mathrm{eV}$. This difference is due to 
the different phases of $\mathrm{LiC}_{12}$ the respective data sets cover. For $\mathrm{LiC}_{16}$ and the disordered phase of $\mathrm{LiC}_{6}$ a common $E_{\mathrm{A}}$ may be established which is close to that of the disordered phase of $\mathrm{LiC}_{12}$.

Comparison with computer simulations of the $\mathrm{Li}^{+}$ migration confirms the $2 \mathrm{D}$ nature of the motion and consequently the crucial role of in-plane order in diffusion.

[1] Graphite Intercalation Compounds I \& II, ed. by H. Zabel and S. A. Solin, Springer, Berlin 1990 and 1992.

[2] Progress in Intercalation Research, ed. by W. MüllerWarmuth and R. Schöllhorn, Kluwer Academic Publishers, Dordrecht 1994.

[3] K. Brandt, Solid State Ionics 69, 173 (1994).

[4] A. Hérold, Synth. Met. 23, 27 (1988).

[5] K. C. Woo, W. A. Kamitakahara, D. P. DiVincenzo, D. S. Robinson, H. Mertwoy, J.W. Mulliken, and J. E. Fischer, Phys. Rev. Lett. 50, 182 (1983).

[6] J. E. Fischer and H. J. Kim, Phys. Rev. B 35, 3295 (1987).

[7] D. S. Robinson and M. B. Salamon, Phys. Rev. Lett. 48, 156 (1982).

[8] J. Rossat-Mignod, A. Wiedenmann, K. C. Woo, J.W. Mulliken, and J. E. Fischer, Solid State Commun. 44, 1339 (1982)

[9] W. Müller-Warmuth in [2], p. 399.

[10] H. Ackermann, P. Heitjans, and H.-J. Stöckmann in: Hyperfine Interactions of Radioactive Nuclei, ed. by J. Christiansen, Topics in Current Physics, Vol. 31, Springer, Berlin 1983, p. 261.

[11] P. Heitjans, Solid State Ionics 18/19, 50 (1986).

[12] P. Heitjans, W. Faber, and A. Schirmer, J. Non-Cryst. Solids 131-133, 1053 (1991).

[13] H. Zabel, A. Magerl, J. J. Rush, and M. E. Meisenheimer, Phys. Rev. B 40, 7616 (1989).

[14] P. Freiländer, P. Heitjans, H. Ackermann, B. Bader, G. Kiese, A. Schirmer, H.-J. Stöckmann, C. Van der Marel, A. Magerl, and H. Zabel, Z. Phys. Chem. N.F. 151, 93 (1987).

[15] A. Schirmer, P. Heitjans, W. Faber, and J. E. Fischer, Synth. Met. 34, 589 (1989).

[16] see e.g. M. Mehring in: Low-Dimensional Conductors and Superconductors, ed. by D. Jerome and L. G. Caron, Plenum Publ. Corp., 1987, p. 185.

[17] I. Burmester, Diplomarbeit, Universität Hannover 1991.

[18] P. Lauginie, M. Letellier, H. Estrade, J. Conard, and D. Guerard, in: Proc. of the Vth London Int. Carbon and Graphite Conf., Ed. Soc. Chem. Ind. 1977, p. 645.

\section{Acknowledgement}

Parts of the work summarized here were performed in various collaborations, especially with $\mathrm{H}$. Ackermann, J. E. Fischer, P. Freiländer, A. Magerl, and H. Zabel. The work was financed by the Bundesministerium für Forschung und Technologie (03-HE2HAN).

[19] N. Bloembergen, E. M. Purcell, and R. V. Pound, Phys. Rev. 73, 679 (1948).

[20] W. Franke and P. Heitjans, Ber. Bunsenges. Phys. Chem. 96, 1674 (1992).

[21] P. Lauginie, H. Estrade-Szwarckopf, and J. Conard, Materials Science Forum 91-93, 545 (1992).

[22] P. Heitjans, Synth. Met. 23, 257 (1988).

[23] D. P. DiVincenzo, C. D. Fuerst, and J. E. Fischer, Phys. Rev. B 29, 1115 (1984).

[24] C. A. Sholl, J. Phys. C 14, 447 (1981).

[25] A. Avogadro and M. Villa, J. Chem. Phys. 66, 2359 (1977).

[26] W. Küchler, P. Heitjans, A. Payer, and R. Schöllhorn, Solid State Ionics 70/71, 434 (1994).

[27] A. Schirmer, P. Heitjans, W. Faber, and J. E. Fischer, Materials Science Forum 91-93, 589 (1992).

[28] J. H. van Vleck, Phys. Rev. 74, 1168 (1948).

[29] T. K. Halsteadt, K. Metcalfe, and T. C. Jones, J. Magn. Reson. 47, 292 (1982).

[30] J. L. Bjorkstam and M. Villa, Magn. Res. Rev. 6, 1 (1980).

[31] M. Meyer, P. Maass, and A. Bunde, Phys. Rev. Lett. 71, 576 (1993)

[32] A. Schirmer, J. E. Fischer, P. Heitjans, H. J. Kim, A. Magerl, D. Vaknin, and H. Zabel, Mol. Cryst. Liq. Cryst. 244, 299 (1994).

[33] N. Daumas and A. Hérold, C.R. Acad. Sci. Paris C268, 373 (1969).

[34] H. J. Kim, A. Magerl, J. E. Fischer, D. Vaknin, P. Heitjans, and A. Schirmer, in: Chemical Physics of Intercalation II, ed. by P. Bernier, J. E. Fischer, S. Roth, and S. A. Solin, NATO ASI B 305, Plenum Press, New York 1993, p. 355

[35] A. Magerl, H. Zabel, and I. S. Anderson, Phys. Rev. Lett. 55, 222 (1985).

[36] J. Corish, F. Jugie, D. A. Morton-Blake, M. Leslie, and F. Bénière, Phys. Rev. B 41, 10870 (1990).

[37] W. Lehnert, W. Schmickler, and A. Bannerjee, Chem. Phys. 163, 331 (1992). 